www.mdpi.com/journal/sensors

Conference Proceedings Paper-Sensors and Applications

\title{
New Design of an Acoustic Array Calibrator for Underwater Neutrino Telescopes
}

\author{
M. Saldaña *, C.D Llorens, I. Felis, J.A. Martínez-Mora and M. Ardid \\ Institut d'Investigació per a la Gestió Integrada de les Zones Costaneres (IGIC), Universitat \\ Politècnica de València (UPV), 46730 Gandia, València, España; \\ E-Mails: cdavid@upv.es (C.D.L.); ivfeen@upv.es (I.F.); jmmora@fis.upv.es (J.A.M.-M.); \\ mardid@fis.upv.es (M.A.) \\ * Author to whom correspondence should be addressed; E-Mail: masalcos@upv.es; \\ Tel.: +34-96-387-7000 (ext. 43681).
}

Published: 11 November 2015

\begin{abstract}
A short bipolar pressure pulse with 'pancake' directivity is produced and propagated when an Ultra-High Energy (UHE) neutrino interacts with a nucleus in water. Nowadays, several acoustic sensors are deployed in deep sea trying to detect the phenomena as first step to build a neutrino telescope. In order to study its feasibility, it is critical to have a calibrator able to mimic the neutrino 'signature'. In previous works we developed a first compact array calibrator prototype that validated the possibility of using the acoustic parametric technique for this aim, but due to limitations in terms of power efficiency, transducer properties and electronics matching, the application was not fully fulfilled. In this paper, we describe a new proposed design of a compact calibrator composed of an array of piezo ceramic tube transducers emitting in axial direction, and new specific electronics adapted to the transducers to feed it more efficiently. The array is operated at high-frequency and, by means of the parametric effect, the emission of the lowfrequency acoustic bipolar pulse is generated permitting to mimic the UHE neutrino acoustic pulse with the required power. All the design processes involved are described and the ceramic characterization tests and results are presented.
\end{abstract}

Keywords: acoustic calibrator; array transducers; UHE neutrinos; acoustic detection; underwater neutrino telescopes; parametric technique 


\section{Introduction}

Ultra High Energy (UHE) neutrinos have become of high interest for the study of ultra-high-energy cosmic rays sources and to test fundamental physics due to the advantages of being stable and having weak interaction with matter. Optical Cherenkov light, radio or acoustic wave techniques have been proposed to detect the neutrino induced energy deposition in water, ice or salt.

Acoustic detection of UHE neutrinos is based on the thermo-acoustic effect [1]. The energy deposition arisen when a neutrino interacts with nuclei in water is constituted in a volume of a few centimetres in radius and several meters in length. This phenomenon induces a local heating in a very short period of time leading to a short pressure pulse signal with bipolar shape and very directive pattern (pancake). The bipolar pulse is emitted from the perpendicular plane of the shower axis.

The feasibility of the acoustic detection neutrino technique is currently under study [2,3] and could be implemented in a new optical-based deep-sea neutrino telescope under construction, the KM3NeT telescope [4] which will have a volume of several cubic kilometres, . and the acoustic detection as a possible and promising technique to cover the detection of Ultra High Energy (UHE) neutrinos, being possible to combine these two neutrino detection techniques for a hybrid underwater neutrino telescopes, especially considering that the optical neutrino detection technique needs acoustic sensors as well for monitoring the position of the optical sensors $[5,6]$. In order to study the viability of the acoustic detection, acoustic transmitters able to imitate the bipolar pulse signal are needed to train and calibrate the sensors, as well as, to improve the classification and discernment of the neutrinos bipolar pulse signals from noise and other background transient signals, which plays a significant mission on acoustic signal detection. Our proposed design, described in this paper, is a compact calibrator composed of an array of piezo-ceramic tube transducers emitting in axial direction with specific electronics adapted to them in order to feed it efficiently. The emission of the low-frequency acoustic bipolar pulse is generated by parametric emission at high-frequency.

\section{Conceptual Design of the Compact Array Calibrator}

The use of parametric acoustic sources to generate neutrino-like signals with cylindrical transducers have been achieved and validated in previous studies [7]. The acoustic parametric effect occurs when two intense monochromatic beams with two close frequencies travel together through the medium. Under these conditions in the region of nonlinear interaction, secondary harmonics of these frequencies are produced at lower frequency with a beam pattern directivity similar to the primary beams. Since this technique offers the advantage of generating a parametric signal much more directive, it allows designing a compact array with fewer units with respect to classical solutions, and thus reducing costs and facilitating the deployment and operation issues. The array calibrator under development will be constituted of few piezo tube ceramics in the same axis-line with an optimal distance separation for obtaining an opening angle of about $1^{\circ}$ and phased emission of the array multielements. The array of transducers will emit in axial direction and produce a bipolar pulse due to the interaction of parametric signals generated for each array element.

On the first phase of the calibrator design, the study and selection of transducers as good candidates for this aim was done. Once the transducers were selected, their characterization was performed and, 
likewise first measures with single transducer emitting parametric neutrino-like signals have been realized. Moreover, in this phase a preliminary study on matching layer materials and transmission loses has been studied. The second phase of the design is finalizing the studies of the parametric emission for single and a group transducers, moulding them, designing the electronics and testing them. The third phase will be the complete integration of the system and the tests at long distances before the in situ test at the detector vicinity.

The array calibrator will be able to work in different operation modes; at linear low frequency range by emitting long non directive signals (easy to detect), at high frequency range by emitting long parametric directive signals, and at high frequency range by emitting the transient and directive parametric bipolar signal. This will allow to use the array transmitter for different functionalities, such as training and tuning the acoustic detector, cross-checking the detector hydrophones, and other marine applications. On the other hand, the different operation modes would allow us to plan a sea campaign plan in order to facilitate the signal detection in the three steps, in increasing difficulty.

\section{Array Calibrator Transducers Characterization}

There are two candidates selected of piezo-ceramic tube transducers for being the emitters of the array calibrator. The reasons of the choice are in terms of resonance frequencies, power emission, dimensions, and costs. Both are able to emit high frequency signals within high power emission and at the low frequencies they have reasonable power levels as well. The first candidate is the piezo-ceramic UCE-534541 with dimensions: outer diameter of $5.3 \mathrm{~cm}$, inner diameter of $4.5 \mathrm{~cm}$ and height of 4.1 $\mathrm{cm}$, its primary frequency of resonance is around $490 \mathrm{kHz}$, but it has also a secondary resonance frequency at low frequency, around $35 \mathrm{kHz}$. The second candidate is the piezo-ceramic UCE-343020 with dimensions: outer diameter of $3.4 \mathrm{~cm}$, inner diameter of $3 \mathrm{~cm}$ and height of $2 \mathrm{~cm}$, its primary frequency of resonance is around $890 \mathrm{kHz}$, but with a secondary frequency resonance around $75 \mathrm{kHz}$. Figure 1 shows the admittance of the UCE-534541 piezo ceramic where the resonance frequencies are.

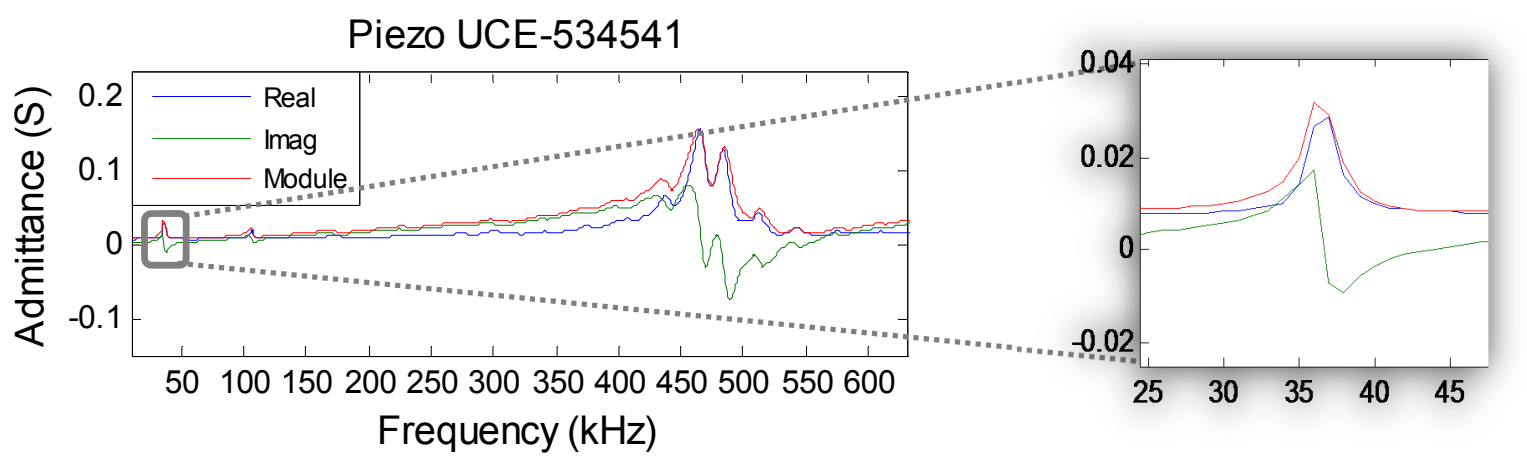

Figure 1. Admittance of the UCE-534541 piezo-ceramic

\subsection{Transmitting Voltage Response and Directivity}

The piezo-ceramics have been characterized both at high and low resonance frequencies in a water tank of $87.5 \times 113 \times 56.5 \mathrm{~cm} 3$ with fresh-water at the laboratory. The calibration of the ceramics has been performed in terms of measuring the Transmitting Voltage Response (TVR) and directivity in emission. The signals sent for the calibration have been tone burst at the different frequency range of 
interest of each transducer. The hydrophones used for the calibration are omnidirectional, the model RESON-TC40348 for the high frequency and the model RESON-TC4034 for low frequency. Figure 2 shows the characterization of both ceramics, (a) shows the sensitivity obtained at high frequency with resonance frequency $\left(F_{R}\right)$ of $490 \mathrm{kHz}$ for the UCE-534541 and $890 \mathrm{kHz}$ for the UCE-343020. The TVR of the UCE-534541 is $159 \mathrm{~dB}$ (re $\mu \mathrm{Pa} / \mathrm{V}$ at $1 \mathrm{~m})$ at $\mathrm{F}_{\mathrm{R}}(490 \mathrm{kHz})$ with a directivity of $\pm 5^{\circ}$. At low frequency the TVR oscillates between $132 \mathrm{~dB}-140 \mathrm{~dB}$ (re $\mu \mathrm{Pa} / \mathrm{V}$ at $1 \mathrm{~m}$ ). On the other hand, the TVR of the UCE-343020 is $162 \mathrm{~dB}($ re $\mu \mathrm{Pa} / \mathrm{V}$ at $1 \mathrm{~m})$ at $\mathrm{F}_{\mathrm{R}}(890 \mathrm{kHz})$ with a directivity of $\pm 7^{\circ}$. The TVR at low frequency oscillates between $132 \mathrm{~dB}-143 \mathrm{~dB}$ (re $\mu \mathrm{Pa} / \mathrm{V}$ at $1 \mathrm{~m})$.

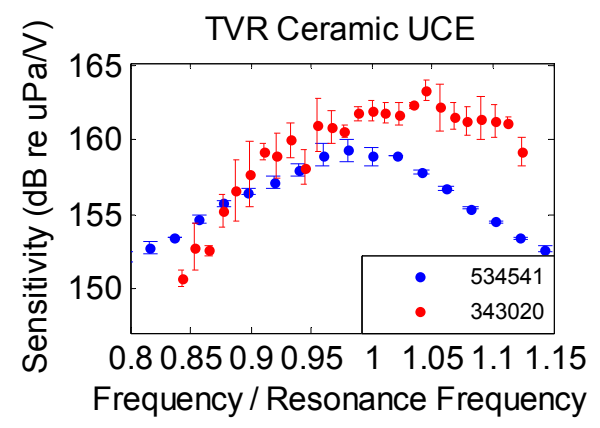

(a)

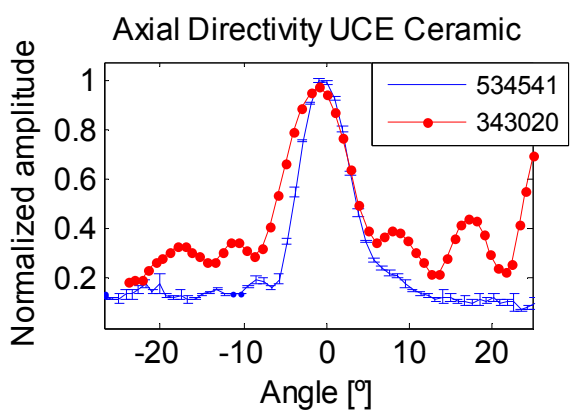

(b)

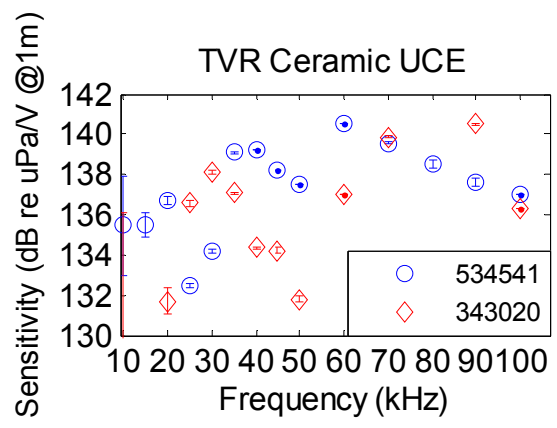

(c)

Figure 2. Characterization of the ceramics and UCE-343020 (a) TVR of UCE-534541 at $\mathrm{F}_{\mathrm{R}}=490 \mathrm{kHz}$ (blue points) and for UCE-343020 at $\mathrm{F}_{\mathrm{R}}=890 \mathrm{kHz}$ (red points) (b) Directivity at $490 \mathrm{kHz}$ for the UCE-534541 (blue line) and at $890 \mathrm{kHz}$ for UCE-343020 (red line)

(c) TVR from $10 \mathrm{kHz}-100 \mathrm{kHz}$

\subsection{Matching Layer}

Two interesting materials for moulding underwater transducers have been acoustically studied in order to know the effect on the signal emitted. The reasons of moulding the nude ceramic are to ensure its protection and holding, to isolate it and match the impedance of the piezo-ceramic to the impedance media. This first study has been applied to a different ceramic, a disc piezo-ceramic of $20 \mathrm{~mm}$ of diameter and $3 \mathrm{~mm}$ of thickness with the resonance frequency at $664 \mathrm{kHz}$. The matching materials under study are the resin RoyaPox 511 and the Polyurethane EL241F, both have interesting acoustic properties for matching the ceramic impedance to the media; acoustic impedance, density and sound speed. The matching layer thickness implemented has been derived to accomplish $\lambda / 4$ of the wave length emitted at the ceramic resonance frequency. The ceramic has been covered with both matching materials, and the TVR and directivity have been determined for the nude ceramic and the two moulded ceramics. The results show the ceramic moulded with the RoyaPox511 resin does not lose sensitivity in the resonance frequency and the directivity curve is very similar to that obtained with nude ceramic, even it seems to reduce the amplitude of lateral lobes. For the case of the ceramic moulded with the EL241F polyurethane there is a sensitivity loss of $5 \mathrm{~dB}$ approximately in the resonant frequency, the directivity does not have a significant variation, it is observed that the main lobe is on the one hand widened slightly. Anyway, the moulding effect on the parametric emission will be studied for both materials as well with the cylindrical ceramics for a final choice. 


\section{First Studies of the Parametric Bipolar Pulse Emission}

The experiment has been carried out in the same water tank as described in section 3.1. The piezoceramic was connected to a linear $55 \mathrm{~dB}$ RF amplifier ENI 1040L to feed the emitter and generate a more powerful signal in order to achieve the non-linear parametric effect. The chosen receiver was the RESON-TC4034, this transducer is an omnidirectional, broad-band hydrophone with enough sensitive to detect the primary beam (high frequency) and be sensitive to the low frequency for the bipolar pulse detection, additionally it was connected to a charge amplifier CCA 1000 (Teledyne RESONC) which amplifies the received signal, especially the low frequency range. This fist study has been performed with the UCE-534541 ceramic, hence, the signal generated for emission has been performed with the modulation at $490 \mathrm{kHz}$. The emitter and the receiver were placed in a distance of $35 \mathrm{~cm}$.

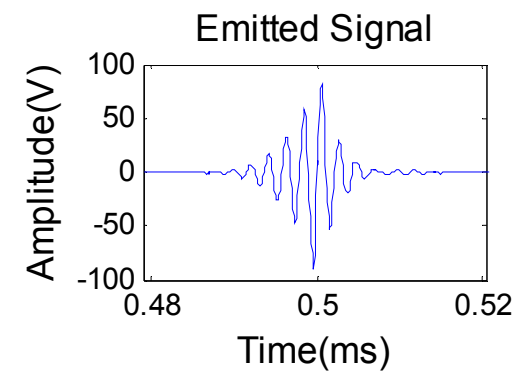

(a)

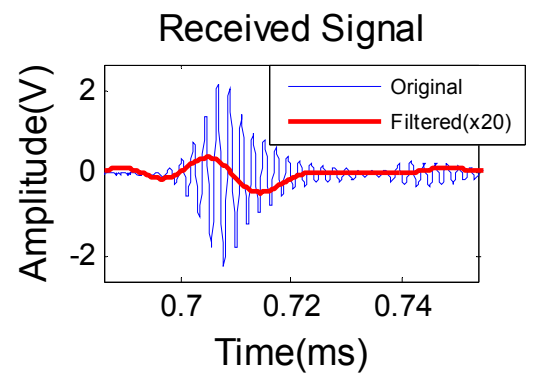

(b)

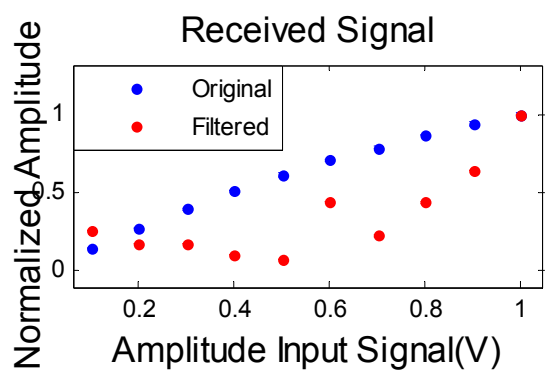

(c)

Figure 3. (a) Emitted Signal (b) Received Signal (blue line) and bipolar signal obtained after applying a low-pass filter (red line) (c) Amplitude of the received (blue line) and filtered signal (red line)

Figure 3 (a) shows the $490 \mathrm{kHz}$ shape-modulated signal emitted, Figure 3 (b) shows the received signal, the primary signal (original) is seen in blue line, the red line is the low band pass frequency filtered received signal (parametric), where the bipolar signal shape is clearly seen. For distinguishing the secondary primary beam a band pass $([5-80] \mathrm{kHz})$ filter has been applied. In order to make sure that the secondary beam has a non-linear behaviour, the amplitude of the parametric signal, as a function of the voltage applied to the transducer, was measured. Figure 3 (c) shows the amplitude behaviour of the received signal, without filtering (original) and with the low-pass filtering (the parametric bipolar signal, secondary beam). Linear behaviour is observable for the amplitude of the primary beam, whereas, for the secondary received signal (parametric bipolar signal) the amplitude behaviour is proportional to the square of the amplitude of the input signal, showing the nonlinearity of the effect.

\section{Conclusions and Future steps}

The results obtained on the piezo-ceramics characterization show that both could be good candidates for being part of the future calibrator since they have an optimal sensitivity in both at high and low frequency and narrow beam directivity at the high frequency which will lead to a tight lowfrequency (parametric) directivity. The matching layer studies validate the materials for the ceramics 
covering. Moreover, the first studies on the acoustic neutrino-like signals generation by using the parametric technic show that the signal reproduction is achieved.

The near future work will consist in completing the studies of the parametric emission for single and group of transducers, and moulding them. In addition, the electronics design will be finalized and tested. After this, the complete integration of the system and the tests at long distances will be performed before the sea campaign test.

\section{Acknowledgments}

We acknowledge the financial support of the Spanish Ministerio de Economía y Competitividad (MINECO) and Ministerio de Ciencia e Innovación (MICINN), Grants FPA2012-37528-C02-02, and Consolider MultiDark CSD2009-00064, of the Generalitat Valenciana, Grants ACOMP/2015/175 PrometeoII/2014/079 and of the European FEDER funds.

\section{Author Contributions}

M. Saldaña and I. Felis have performed the tests and analyzed the data. C. Llorens is designing the electronics for the calibrator and J.A. Martínez-Mora and M. Ardid have proposed the calibrator design and supervised the different tasks involved and the results.

\section{Conflicts of Interest}

The authors declare no conflict of interest.

\section{References}

1 G.A.Askaryan. Hydrodynamical emission of tracks of ionising particles in stable liquids. J. A Energy 3 (1957) 921.

2 J.A. Aguilar, et al., AMADEUS, The acoustic neutrino detection test system of the ANTARES deep-sea neutrino telescope, Nucl. Instr. and Meth. A 2011, 626, 128-143.

3 G. Riccobene (for the NEMO Collaboration), Long-term measurements of acoustic background noise in very deep sea, Nucl. Instrum. Meth. A 2009, 604, S149.

4 The KM3NeT Collaboration, KM3NeT Technical Design Report (2010) ISBN 978-90-6488-0339, available on www.km3net.org.

5 M. Ardid, Calibration in acoustic detection of neutrinos. Nucl. Instr. and Meth. A 2009, 604, S203.

6 M. Ardid, Positioning system of ANTARES telescope. Nucl. Instr. and Meth. A 2009, 602, 174.

7 M. Ardid et al., Acoustic Transmitters for Underwater Neutrino Telescopes, Sensors, vol. 12 (2012), pp. 4113-4132.

(C) 2015 by the authors; licensee MDPI, Basel, Switzerland. This article is an open access article distributed under the terms and conditions of the Creative Commons Attribution license (http://creativecommons.org/licenses/by/4.0/). 